\title{
Reply regarding pediatric salivary gland imaging
}

\author{
Zachary T. Boyd • Lisa H. Lowe • Asha R. Goud • \\ Lei Shao
}

Received: 20 August 2009 / Accepted: 26 August 2009 / Published online: 24 September 2009

(C) Springer-Verlag 2009

Sir,

We thank Gadodia et al. [1] for their interest in our pictorial essay [2] and for clarifying the definition of accessory parotid gland tissue. We agree with their comment and would also like to reiterate the take-home point regarding accessory parotid tissue, which, given its high predominance, should not be mistaken for a mass.

Regarding the comment on our omission of chronic recurrent parotitis, we did so because it is not typically imaged and often managed on a clinical basis. However, the interesting points made by Gadodia et al. [1] regarding chronic recurrent parotitis are notable, and it is certainly a diagnosis with which one should be familiar.

\section{References}

1. Gadodia A, Seith A, Sharma R (2009) Pediatric salivary gland imaging. Pediatr Radiol 39 doi:10.1007/s00247-009-1408-1

2. Boyd ZT, Goud AR, Lowe LH et al (2009) Pediatric salivary gland imaging. Pediatr Radiol 39:710-722. doi:10.1007/s00247-0091232-7

Z. T. Boyd $(\bowtie)$

Department of Medicine, University of Missouri-Kansas City,

2411 Holmes St.,

Kansas City, MO 64108, USA

e-mail: Zachary.boyd@gmail.com

L. H. Lowe

Department of Radiology, Children's Mercy Hospitals and Clinics and the University of Missouri-Kansas City,

Kansas City, MO, USA

\section{A. R. Goud}

Department of Radiology,

University of California Irvine Medical Center,

Orange, CA, USA

L. Shao

Department of Pathology, Children's Mercy Hospitals and Clinics and the University of Missouri-Kansas City,

Kansas City, MO, USA 